\title{
RECENT RESULTS IN CHIRAL NUCLEAR DYNAMICS
}

\author{
ULF-G. MEIßNER \\ Universität Bonn, Helmholtz-Institut für Strahlen- und Kernphysik (Theorie) \\ Nußallee 14-16, D-53115 Bonn, Germany \\ E-mail: meissner@itkp.uni-bonn.de \\ VÉRONIQUE BERNARD \\ Laboratoire de Physique Théorique, Université Louis Pasteur \\ 3-5, rue de l'Université, F-67084 Strasbourg, France \\ EVGENY EPELBAUM, WALTER GLÖCKLE \\ Ruhr-Universität Bochum, Institut für Theoretische Physik II, \\ Universitätsstraße 150, D-44870 Bochum, Germany
}

\begin{abstract}
Some recent developments in the description of nuclear forces and few-nucleon
\end{abstract} dynamics derived from chiral effective field theory are reviewed.

\section{Introduction}

Nuclear forces can be systematically analyzed in the framework of chiral effective field theory (EFT). The starting point is a chiral Lagrangian of pions, nucleons and external sources, like e.g. photons. At low energies, one can expand S-matrix elements and transition currents in powers of small external momenta/energies and the light quark masses. In case of two (or more) nucleons, an additional non-perturbative resummation is necessary to generate the large S-wave scattering lengths or small nuclear binding energies. This is most efficiently done by applying a chiral power counting scheme to the nucleon-nucleon (NN) potential. With that, one solves the corresponding Lippmann-Schwinger (LS) equation for bound and scattering states ${ }^{1}$, employing a regularization procedure consistent with the underlying symmetries. This framework allows for a systematic evaluation of the forces between 2,3 , or 4 nucleons, the gauge-invariant coupling of external probes or the investigation of nuclear physics in the limit of vanishing quark masses. In what follows, I will briefly address some of 

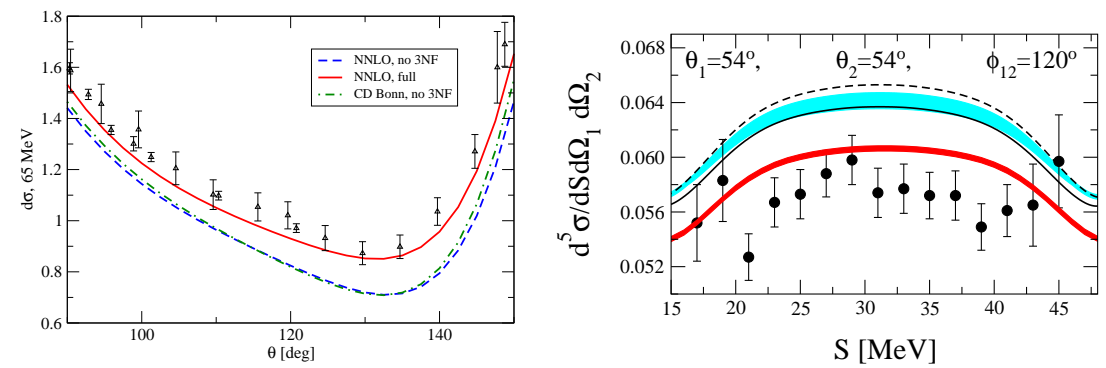

Figure 1. Left: Differential cross section for $n d$ scattering at $65 \mathrm{MeV}$. Solid (dashed) line: NNLO prediction with (without) 3NF. The dot-dashed line gives the prediction based on the CD-Bonn potential without 3NF. Right: $p d$ breakup cross section data in $\left[\mathrm{mb} \mathrm{MeV}^{-1} \mathrm{sr}^{-2}\right]$ along the kinematical locus $\mathrm{S}$ (in $\mathrm{MeV}$ ) at $65 \mathrm{MeV}$. The data are from ${ }^{6}$. NNLO predictions (dark shaded band) compared to the conventional NN forces+TM $3 \mathrm{NF}$ predictions (light shaded band). The solid (dashed) line refers to the AV18+URBANA IX (CD Bonn+TM') results.

these issues. Space forbids to account for all the interesting work done by many researchers and references are only given that are of direct relevance to the topic under consideration.

\section{Few-nucleon systems}

The two-nucleon force can be well described within this framework, see e.g. ${ }^{2}$. More interesting is the three-nucleon force (3NF), which has been one of the most sought after objects in nuclear physics. In chiral EFT, it first appears at next-to-leading order (NLO), but these contributions can be shown to vanish ${ }^{1,3,4}$. At NNLO, there are three different topologies: two-pion-exchange between 3 nucleon lines, one-pion-exchange between a $4 \mathrm{~N}$ contact term and a nucleon line and a $6 \mathrm{~N}$ contact interaction. Two low-energy constants (LECs) (related to the latter two topologies) appear. These can be fixed from the triton binding energy (BE) and the $n d$ doublet scattering length. With that, the $3 \mathrm{NF}$ is completely determined and the properties of few-nucleon systems can be calculated in a parameter-free manner ${ }^{5}$. In most cases, the predictions based on chiral EFT are very close to the ones based on more conventional approaches, that is the socalled high precision NN potentials combined with some (non-chiral) 3NF, see e.g. the differential cross section for elastic $n d$ scattering at $65 \mathrm{MeV}$ (left panel of Fig. 1). There are, however, exceptions, see the specific breakup configuration in the right panel of Fig. 1. This is indeed the break-up observable where the largest deviations of the two approaches are observed. 
Solving the pertinent Faddeev-Yakubovsky equations for the 4N system, one can calculate e.g. the BE of ${ }^{4} \mathrm{He}$. We find ${ }^{5}$

$$
\mathrm{BE}\left({ }^{4} \mathrm{He}\right)=29.51 \ldots 29.98 \mathrm{MeV} \text { for } \Lambda=500 \ldots 600 \mathrm{MeV} \text {, }
$$

where $\Lambda$ is the cut-off in the regularized LS equation. The empirical value (corrected for isospin breaking effects) is $29.8 \pm 0.1 \mathrm{MeV}$. Thus there is very little room for a $4 \mathrm{NF}$. Interestingly, the first corrections to the chiral $3 \mathrm{NF}$ are free of $6 \mathrm{~N}$ contact LECs and are presently worked out.

\section{Nuclear forces in the chiral limit}

Because of the smallness of the up and down quark masses, one does not expect significant changes in systems of pions or pions and one nucleon when the quark masses are set to zero (with the exception of well understood chiral singularities like e.g. in the pion radius or the nucleon polarizabilities). The situation is more complicated for systems of two (or more) nucleons, as first discussed in EFT in ${ }^{7}$. Here, I report on similar work ${ }^{8}$ that is mostly concerned with the properties of the deuteron and the S-wave scattering lengths as a function of the quark (pion) mass. These questions are not only of academic interest, but also of practical use for interpolating results from lattice gauge theory. E.g. the S-wave scattering lengths have been calculated on the lattice using the quenched approximation ${ }^{9}$. Another interesting application is related to imposing bounds on the time-dependence of some fundamental coupling constants from the NN sector, as discussed in ${ }^{10}$. At NLO the following contributions have to be accounted for (in addition to the LO OPE and contact terms without derivatives): i) contact terms with two derivatives or one $M_{\pi}^{2}$-insertion, ii) renormalization of the OPE, iii) renormalization of the contact terms, and iv) two-pion exchange (TPE). This induces explicit and implicit quark mass dependences. In the first category fall the pion propagator that becomes Coulomb-like in the chiral limit or the $M_{\pi}^{2}$ corrections to the leading contact terms. These are parameterized by the LECs $\bar{D}_{S, T}$ at NLO. These LECs can at present only be estimated using dimensional analysis and resonance saturation ${ }^{11}$. The implicit pion mass dependence enters at NLO through the pion-nucleon coupling constant, ${ }^{\mathrm{a}}$ expressed through the pion mass dependence of $g_{A} / F_{\pi}$,

$$
\Delta=\left(\frac{g_{A}^{2}}{16 \pi^{2} F_{\pi}^{2}}-\frac{4}{g_{A}} \bar{d}_{16}+\frac{1}{16 \pi^{2} F_{\pi}^{2}} \bar{l}_{4}\right)\left(M_{\pi}^{2}-\tilde{M}_{\pi}^{2}\right)-\frac{g_{A}^{2} \tilde{M}_{\pi}^{2}}{4 \pi^{2} F_{\pi}^{2}} \ln \frac{\tilde{M}_{\pi}}{M_{\pi}} .
$$

\footnotetext{
${ }^{a}$ Note that the quark mass dependence of the nucleon mass only enters at NNLO.
} 


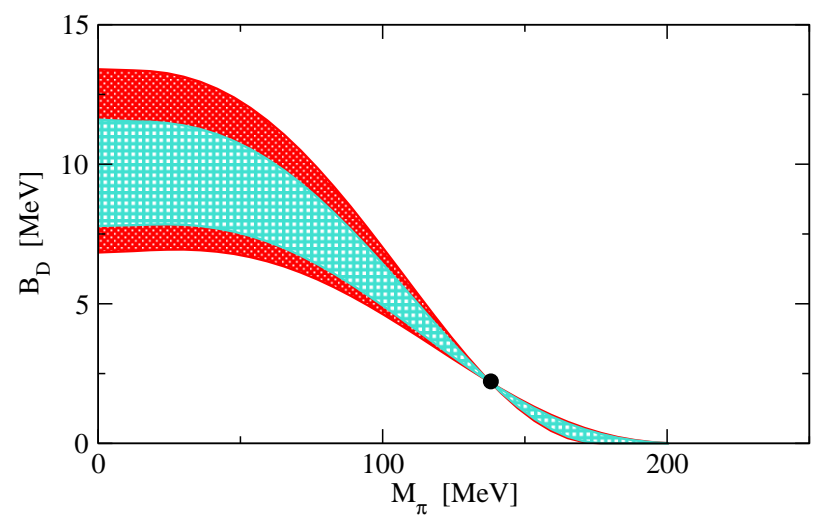

Figure 2. Deuteron BE versus $\tilde{M}_{\pi}$. The shaded areas show allowed values. The light shaded band corresponds to our main result with the uncertainty due to the unknown LECs $\bar{D}_{S, T}$. The dark shaded band gives the additional uncertainty due to the uncertainty of $\bar{d}_{16}$. The heavy dot shows the BE for the physical case $\tilde{M}_{\pi}=M_{\pi}$.

Here $\bar{l}_{4}, \bar{d}_{18}$ and $\bar{d}_{16}$ are LECs related to pion and pion-nucleon interactions, and the value of the pion mass is denoted by $\tilde{M}_{\pi}$ in order to distinguish it from the physical one denoted by $M_{\pi}$. In particular, $\bar{d}_{16}$ has been determined in various fits to describe $\pi N \rightarrow \pi \pi N$ data, see ${ }^{12}$.

The deuteron BE as a function of the pion mass is shown in Fig.2, we find that the deuteron is stronger bound in the chiral limit than in the real world with the $\mathrm{BE} B_{\mathrm{D}}^{\mathrm{CL}}=9.6 \pm 1.9_{-1.0}^{+1.8} \mathrm{MeV}$, where the the first indicated error refers to the uncertainty in the value of $\bar{D}_{3 S_{1}}$ and $\bar{d}_{16}$ being set to its average value while the second indicated error shows the additional uncertainty due to the uncertainty in the determination of $\bar{d}_{16}$ We find no other bound states, although the higher $\mathrm{S}=1$ partial waves rise linear with momentum due to the Coulomb-like pion propagator. Other deuteron properties are given in the table. We note in particular the large uncertainty for the quadrupole moment, which is related to its increased sensitivity to short distance physics. Last but not least, we found smaller (in magnitude) and more natural values for the two $\mathrm{S}$-wave scattering lengths in the chiral limit, $a_{\mathrm{CL}}\left({ }^{1} S_{0}\right)=-4.1 \pm 1.6_{-0.4}^{+0.0} \mathrm{fm}$, and $a_{\mathrm{CL}}\left({ }^{3} S_{1}\right)=1.5 \pm 0.4_{-0.3}^{+0.2} \mathrm{fm}$. As stressed in ${ }^{8}$, one needs lattice data for pion masses below $200 \mathrm{MeV}$ to perform a stable interpolation to the physical value of $M_{\pi}$. We conclude that nuclear physics in the chiral limit is much more natural than in the real world. 
Table 1. Deuteron properties at NLO compared to the data.

\begin{tabular}{|c|c|c|c|}
\hline & physical case & chiral limit & experiment \\
\hline$B_{\mathrm{D}}[\mathrm{MeV}]$ & 2.17 & $9.6 \pm 1.9_{-1.0}^{+1.8}$ & $2.22456612(12)$ \\
$Q_{\mathrm{D}}\left[\mathrm{fm}^{2}\right]$ & 0.274 & $0.247 \pm 0.030_{-0.005}^{+0.008}$ & $0.2859(3)$ \\
$r_{\mathrm{D}}[\mathrm{fm}]$ & 1.975 & $1.266 \pm 0.085_{-0.034}^{+0.044}$ & $1.9671(6)$ \\
$\mu_{\mathrm{D}}[\mathrm{n} . \mathrm{m}]$. & 0.860 & $0.820 \pm 0.002_{-0.006}^{+0.003}$ & $0.8574382284(94)$ \\
\hline
\end{tabular}

\section{Pion-deuteron scattering}

The pion-nucleon $(\pi \mathrm{N})$ S-wave scattering lengths are quantities of fundamental importance in hadronic physics. They provide an important test of QCD symmetries and the pattern of their breaking. They also provide a crucial constraint on the $\pi \mathrm{N}$ interaction and, as such, have an impact on our understanding of nucleon-nucleon $(\mathrm{NN})$ scattering, the three-nucleon force, and pion-nucleus scattering. They can be e.g. determined by analyzing low energy scattering data making use of chiral perturbation theory, for the most recent analysis in the isospin limit see ${ }^{13}$. However, a novel evaluation of electromagnetic corrections has cast some doubt on the existing phase shift analyses ${ }^{14}$. An independent approach to the $\pi \mathrm{N}$ scattering lengths involves analyzing pionic-atom level shifts and widths. In the Coulombic $\pi^{-}$-p system, the strong-interaction shift in the energy (the width) can be used to infer a value for $a^{-}+a^{+}\left(a^{-}\right)$. Here, $a^{-}$ $\left(a^{+}\right)$refers to the isovector (isoscalar) scattering length. Thus, neither $\pi \mathrm{N}$ scattering nor the $\pi^{-}-\mathrm{p}$ atom provide a strong constraint on $a^{+}$. The isoscalar scattering length may well be probed more directly in the $\pi^{-}$-d atom. The most recent measurement ${ }^{15}$ of the $\pi$-d atomic-level shift yields: $a_{\pi d}=(-0.0261 \pm 0.0005)+i(0.0063 \pm 0.0007) M_{\pi}^{-1}$ for the $\pi$-d scattering length - a measurement that is remarkably accurate given the usual level of precision in hadronic-physics experiments. In order to precisely relate this number to $a^{+}$an EFT of threshold $\pi$-d scattering is required. In ${ }^{16}$ we have formulated a novel power counting which accounts for the fact that the deuteron binding momentum $\gamma \simeq 45 \mathrm{MeV}$ is sizeably smaller than the pion mass ${ }^{17}$. This lets one isolate easily the dominant contributions and calculate the corrections. To fourth order, one gets for the $\pi$-d scattering length,

$$
\operatorname{Re} a_{\pi d}=2 \frac{(1+\mu)}{(1+\mu / 2)}\left(a^{+}+(1+\mu)\left[\left(a^{+}\right)^{2}-2\left(a^{-}\right)^{2}\right] \frac{1}{2 \pi^{2}}\left\langle\frac{1}{\vec{q}^{2}}\right\rangle_{\mathrm{wf}}\right.
$$




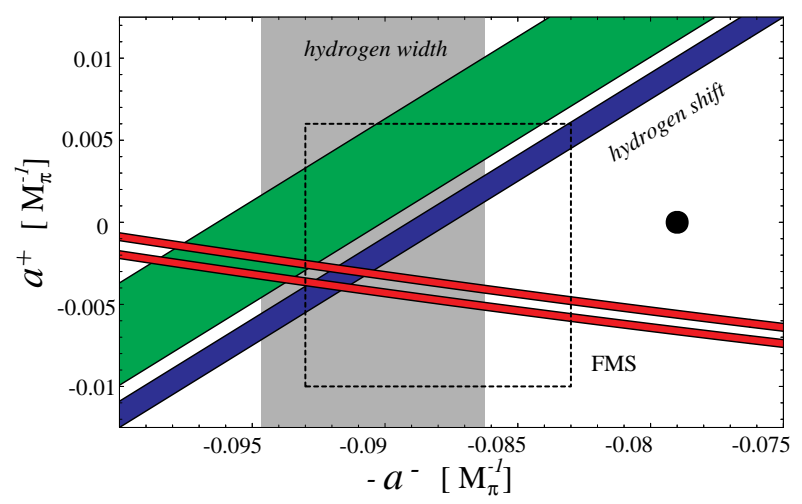

Figure 3. Plot of $a^{+}$vs $-a^{-}$. The light shaded region and the dark band are from the experimental pionic-hydrogen width and shift, respectively, taken from Ref. ${ }^{19}$. The shaded region above the dark band is the hydrogen shift computed in bound state EFT ${ }^{18}$. The dotted line encompasses the constraints from $\pi$-N phase shift data and is taken from Ref. ${ }^{20}$. The dot is leading order $\chi \mathrm{PT}$ (current algebra). The two parallel bands are from Eq. (3) evaluated with the NLO wavefunction with an ultraviolet cutoff of $400 \mathrm{MeV}$ (upper curve) and $600 \mathrm{MeV}$ (lower curve).

$$
\left.+(1+\mu)^{2}\left[\left(a^{+}\right)^{3}-2\left(a^{-}\right)^{2}\left(a^{+}-a^{-}\right)\right] \frac{1}{4 \pi}\left\langle\frac{1}{|\vec{q}|}\right\rangle_{\mathrm{wf}}\right)+a_{(\text {boost })},
$$

where some higher-order effects are subsumed into the $\pi \mathrm{N}$ scattering lengths, $\mu=M_{\pi} / m_{p} \simeq 1 / 7$ is the small threshold parameter, the brackets \langle\rangle$_{\mathrm{wf}}$ denote a deuteron matrix-element in momentum space, and the boost correction is discussed in ${ }^{16}$. The resulting relationship between $a^{-}$and $a^{+}$ is displayed in Fig. 3. Our model-independent extraction of the $\pi \mathrm{N}$ scattering lengths results from the overlap region in Fig. 3 using the hydrogen shift computed in bound state EFT ${ }^{18}$ (upper curve). We find

$$
a^{-}=0.0936 \pm 0.0011 M_{\pi}^{-1}, a^{+}=-0.0029 \pm 0.0009 M_{\pi}^{-1} .
$$

If one uses instead the model-dependent extraction using the hydrogen shift of Ref. ${ }^{19}$, these numbers change to $a^{-}=0.0917 \pm 0.0013 M_{\pi}^{-1}, a^{+}=$ $-0.0034 \pm 0.0008 M_{\pi}^{-1}$. We note that our result is consistent with the recent work of Ref. ${ }^{21}$. What needs to be done is a systematic inclusion of isospin-breaking corrections in the EFT for the $\pi$-d system.

\section{Neutral pion electroproduction off deuterium}

The last topic I would like to discuss briefly is neutral pion electroproduction off deuterium. While $\pi^{0}$ production off protons has become one 


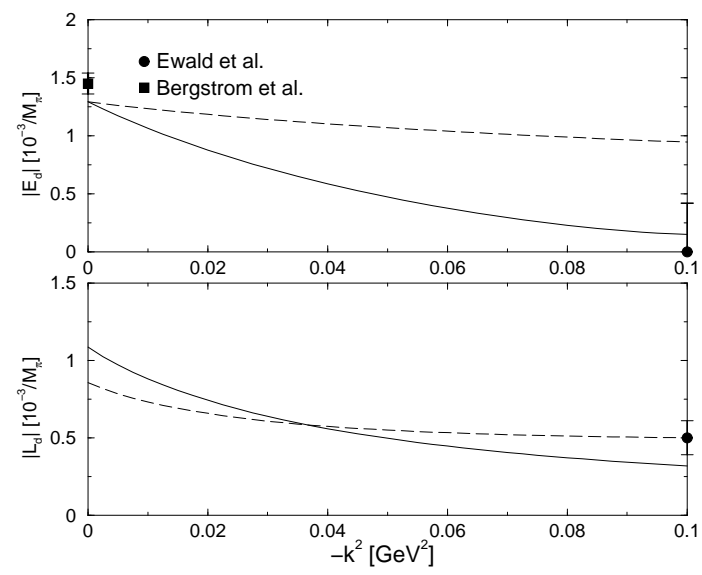

Figure 4. Modulus of the threshold S-wave multipoles $\left|E_{d}\right|$ and $\left|L_{d}\right|$ as a function of the photon virtuality in comparison to the photon point data from SAL ${ }^{23}$ and the electroproduction data from MAMI ${ }^{24}$. The sign of the experimental result for $L_{d}$ is taken to agree with the theoretical prediction. Solid (dashed) lines: Fit 2 (1), as explained in 26 .

of the major testing grounds of chiral $\pi \mathrm{N}$ dynamics, the elusive neutron photo/electroproduction amplitude can only be obtained from experiments on light nuclei. While the photoproduction case $\gamma d \rightarrow \pi^{0} d$ has been quite a success (the chiral prediction of ${ }^{22}$ was verified by the SAL experiment ${ }^{23}$ ), the first measurement of pion electroproduction off deuterium at MAMI ${ }^{24}$ was in stark disagreement with the third order threshold prediction of ${ }^{25}$, in which the single scattering contribution was scaled to the value at the real photon point. This was significantly improved in the above threshold (partial) fourth order calculation of ${ }^{26}$, where the two appearing LECs were determined in two different ways. In addition, we also used deuteron wave functions derived from chiral nuclear EFT. With that, the existing differential cross section data at four different excess energies and three photon polarizations each could be satisfactorily described. Furthermore, the dependence of the transverse and the longitudinal S-wave amplitude on the photon virtuality is now in agreement with the data, see Fig.4 (although some discrepancies remain). The two lines shown in the figure can be considered as a measure of the theoretical uncertainty at this order. Clearly, the calculation presented in ${ }^{26}$ needs to be improved, in particular, the fourth order corrections to the $\mathrm{P}$-waves and the three-body terms have to be included (note that similar work for the $\mathrm{P}$-waves in neutral pion pro- 
duction off protons has only appeared recently ${ }^{27}$ ). This reaction shows nicely the intricate interplay of chiral nucleon and nuclear dynamics.

\section{Acknowledgements}

I am grateful to Koichi Yamawaki for inviting me and to all the organizers for their magnificent job. My collaborators Silas Beane, Hiroyuki Kamada, Hermann Krebs, Andreas Nogga, Daniel Phillips and Henryk Witala are thanked for sharing their insight into the various topics discussed here.

\section{References}

1. S. Weinberg, Nucl. Phys. B363, 3 (1991).

2. E. Epelbaum, W. Glöckle and Ulf-G. Meißner, Nucl. Phys. A671, 295 (2000).

3. U. van Kolck, Phys. Rev. C49, 2932 (1994).

4. E. Epelbaoum, W. Glöckle and Ulf-G. Meißner, Nucl. Phys. A637, 107 (1998).

5. E. Epelbaum, et al., Phys. Rev. C66, 064001 (2002).

6. J. Zejma et al., Phys. Rev. C55, 42 (1997).

7. S.R. Beane, et al., Nucl. Phys. A700, 377 (2002).

8. E. Epelbaum, Ulf-G. Meißner, and W. Glöckle, Nucl. Phys. A714, 535 (2003); nucl-th/0208040

9. M. Fukugita, et al., Phys. Rev. D52, 3003 (1995).

10. S.R. Beane and M.J. Savage, Nucl. Phys. A713, 148 (2003).

11. E. Epelbaum, Ulf-G. Meißner, W. Glöckle, and Ch. Elster, Phys. Rev. C65, 044001 (2002).

12. N. Fettes, V. Bernard, and Ulf-G. Meißner, Nucl. Phys. A699, 269 (2000);

N. Fettes, doctoral thesis, published in Berichte des Forschungszentrum Jülich, 3814, (2000).

13. N. Fettes and Ulf-G. Meißner, Nucl. Phys. A676, 311 (2000).

14. N. Fettes and Ulf-G. Meißner, Nucl. Phys. A693, 693 (2001).

15. P. Hauser et al., Phys. Rev. C58, 1869 (1998).

16. S.R. Beane et al., hep-ph/0206219.

17. S.R. Beane and M.J. Savage, nucl-th/0204046.

18. J. Gasser et al., Eur. Phys. J. C26, 13 (2002).

19. H.C. Schröder et al., Phys. Lett. B469, 25 (1999); Eur. Phys. J. C21, 473 (2001).

20. N. Fettes, Ulf-G. Meißner, and S. Steininger, Nucl. Phys. A640, 199 (1998).

21. T.E. Ericson, B. Loiseau and A.W. Thomas, Phys. Rev. C66, 014005 (2002).

22. S. R. Beane, V. Bernard, T.-S.H. Lee, Ulf-G. Meißner and U. van Kolck, Nucl. Phys. A618, 381 (1997).

23. J.C. Bergstrom, et al., Phys. Rev.C57, 3203 (1998).

24. I. Ewald et al., Phys. Lett. B499,238 (2001).

25. V. Bernard, H. Krebs and Ulf-G. Meißner, Phys. Rev. C61, 058021 (2000).

26. H. Krebs, V. Bernard, and Ulf-G. Meißner, Nucl. Phys. A713, 405 (2003).

27. V. Bernard, N. Kaiser and Ulf-G. Meißner, Eur. Phys. J. A11, 209 (2001). 